\title{
Theory of the Topological Spin Hall Effect in Antiferromagnetic Skyrmions: Impact on Current-Induced Motion
}

\author{
C. A. Akosa, ${ }^{1,2, *}$ O. A. Tretiakov, ${ }^{3}$ G. Tatara, ${ }^{1,4}$ and A. Manchon ${ }^{2}$ \\ ${ }^{1}$ RIKEN Center for Emergent Matter Science (CEMS), 2-1 Hirosawa, Wako, Saitama 351-0198, Japan \\ ${ }^{2}$ King Abdullah University of Science and Technology (KAUST), Physical Science and Engineering (PSE) Division, \\ Thuwal 23955-6900, Saudi Arabia \\ ${ }^{3}$ Institute for Materials Research, Tohoku University, Sendai 980-8577, Japan \\ ${ }^{4}$ RIKEN Cluster for Pioneering Research (CPR), 2-1 Hirosawa, Wako, Saitama, 351-0198 Japan
}

(Received 5 September 2017; revised manuscript received 11 May 2018; published 30 August 2018)

\begin{abstract}
We demonstrate that the nontrivial magnetic texture of antiferromagnetic Skyrmions (AFM Sks) promotes a nonvanishing topological spin Hall effect (TSHE) on the flowing electrons. This effect results in a substantial enhancement of the nonadiabatic torque and, hence, improves the Skyrmion mobility. This nonadiabatic torque increases when decreasing the Skyrmion size, and, therefore, scaling down results in a much higher torque efficiency. In clean AFM Sks, we find a significant boost of the TSHE close to the van Hove singularity. Interestingly, this effect is enhanced away from the band gap in the presence of nonmagnetic interstitial defects. Furthermore, unlike their ferromagnetic counterpart, the TSHE in AFM Sks increases with an increase in the disorder strength, thus opening promising avenues for materials engineering of this effect.
\end{abstract}

DOI: 10.1103/PhysRevLett.121.097204

Introduction.-As the spintronics community advances the search for high-efficiency, high-density, and low-powerconsuming spintronic devices, alternative materials other than conventional ferromagnets (FMs) are being continuously introduced and explored. Besides FMs, antiferromagnets (AFMs) have recently drawn significant attention [1,2]. The experimental observation of bulk spin-orbit torques (SOTs) in locally inversion asymmetric CuMnAs [3], the demonstration of AFM-assisted zero-field SOT switching [4,5], and the achievement of large anomalous and spin Hall effects in noncollinear AFMs [6-8] open promising perspectives for the implementation of AFMs into efficient spin devices. The latter effect is particularly intriguing, since it emerges from the coexistence of spin-orbit coupling (SOC)driven Berry curvature and noncollinear magnetism. In addition, it has also been predicted that AFM textures such as domain walls driven by SOTs can move much faster than their FM counterparts due to the absence of Walker breakdown $[9,10]$. Therefore, the interplay between topological spin transport and the dynamics of AFM textures is a promising route to explore towards the realization of efficient current-driven control of the AFM order parameter.

Recently, ferromagnetic Skyrmions (FM Sks) have been proposed as good candidates for technological applications due to their weak sensitivity to defects [11-13], ultralow critical current density [13-19], enhanced nonadiabatic torque [20,21], and substantial TSHE [22,23]. In spite of these remarkable properties, FM Sks suffer from the socalled Skyrmion Hall effect [19,24,25], a motion transverse to the current flow. This parasitic effect hinders the robust electrical manipulation of FM Sks. In contrast, both the analytical theory and micromagnetic simulations recently showed that, in AFM Sks, the Skyrmion Hall effect vanishes by symmetry [26-30].

In this Letter, we demonstrate that the nontrivial magnetic texture of AFM Sks promotes a nonvanishing TSHE on the flowing electrons. This effect results in a substantial enhancement of the nonadiabatic torque and, hence, improves the Skyrmion mobility. This nonadiabatic torque increases as the Skyrmion size decreases, and, as a result, scaling down results in a much higher torque efficiency. In clean systems, we find a significant enhancement of the TSHE close to the van Hove singularity. Most importantly, unlike FM Sks [23], the TSHE in AFM Sks increases in the presence of nonmagnetic interstitial defects. Moreover, the TSHE is enhanced away from the band gap in the presence of these defects.

Phenomenological model.-Motivated by the prediction of a metastable single AFM Sk on a square lattice [29-32], our analysis begins with an isolated $G$-type (i.e., checkerboard) AFM Sk with equivalent sublattices $a$ and $b$. The conduction electrons are coupled to the Néel order $\mathbf{n}(\boldsymbol{r}, t)$ via an exchange energy $J$. For a smooth and slowly varying Néel order parameter, the emergent electromagnetic fields of the $\eta$ sublattice acting on electrons with spin $\sigma$ are derived as $[33,38-40]$

$$
\begin{aligned}
& \mathbf{E}_{\mathrm{em}}^{\eta, \sigma}=(\sigma \hbar / 2 e) P_{\sigma}^{\eta} \mathcal{N}_{t, i}(\boldsymbol{r}) \mathbf{e}_{i}, \\
& \mathbf{B}_{\mathrm{em}}^{\eta, \sigma}=-(\sigma \hbar / 2 e) P_{\sigma}^{\eta} \mathcal{N}_{x, y}(\boldsymbol{r}) \mathbf{z},
\end{aligned}
$$


where $\mathcal{N}_{\mu, \nu}(\boldsymbol{r})=\left(\partial_{\mu} \mathbf{n} \times \partial_{\nu} \mathbf{n}\right) \cdot \mathbf{n}$, with $\mu, \nu \in(t, x, y)$, $\sigma=+(-) 1$ for $\uparrow(\downarrow)$ spin, $P_{\sigma}^{\eta}=\left(1+\sigma \eta P_{k}\right) / 2$, where $P_{k}=J / \varepsilon_{k}$ is the polarization of the density of state per sublattice, and $\varepsilon_{k}$ is the energy dispersion [40,41]. Notice that $P_{k}$ takes a value of 1 close to the van Hove singularity or as the exchange $J$ goes to infinity. In these limits, the two sublattices behave like two independent parallel ferromagnets $\left(P_{\uparrow}^{a}=1, P_{\downarrow}^{a}=0\right)$ and $\left(P_{\uparrow}^{b}=0, P_{\downarrow}^{b}=1\right)$. Therefore, unlike FM Sks, in which electrons feel an emergent electromagnetic field of opposite sign for different spins, in real AFM Sks (finite $J$ ), the magnitude of this field is both spin- and sublattice-dependent and strongly depends on the dispersion [42].

Our analysis is based on an AFM Sk with radius $r_{0}$, embedded in a large system of radius $R \gg r_{0}$ moving rigidly with velocity $\mathbf{v}\left[\right.$ i.e., $\left.\partial_{t} \mathbf{n}=-(\mathbf{v} \cdot \nabla) \mathbf{n}\right]$. We chose without the loss of generality the profile given as $\mathbf{n}=(\cos \Phi \sin \theta$, $\sin \Phi \sin \theta, \cos \theta)$, where $\cos \theta=p\left(r_{0}^{2}-r^{2}\right) /\left(r_{0}^{2}+r^{2}\right)$ and $\Phi=q \operatorname{Arg}(x+i y)+c \pi / 2$ define the polar and azimuthal angles, respectively. The constants $p, q$, and $c$, which take values of \pm 1 , define the polarization, vorticity, and chirality, respectively [33]. Under the action of an external electric field along the $x$ axis (i.e., $\mathbf{E}=E \mathbf{x}$ ), the local charge and spin current densities per sublattice read [33]

$$
\begin{aligned}
\boldsymbol{j}_{e}^{\eta \eta}= & (1 / 2)\left[\sigma_{0} \mathbf{x}+\eta \sigma_{x y}(\boldsymbol{r}) \mathbf{y}\right] E \\
& +\eta(\hbar / 4) \bar{P}_{0} \sigma_{0} \mathcal{N}_{x, y}(\boldsymbol{r})(\mathbf{v} \times \mathbf{z}), \\
\boldsymbol{j}_{s}^{\eta}= & \mathbf{n} \otimes(1 / 2)\left[\eta \mathbf{x}-p q \beta_{T}(\boldsymbol{r}) \mathbf{y}\right] b_{J} \\
& +(p q / 2) \alpha_{T}(\boldsymbol{r}) \mathbf{n} \otimes(\mathbf{v} \times \mathbf{z}),
\end{aligned}
$$

where $\sigma_{0(H)} / 2$ is the longitudinal (ordinary Hall) conductivity of sublattice $\eta$ and $\sigma_{x y}(\boldsymbol{r})=(\hbar / 2 e) \bar{P}_{H} \sigma_{H} \mathcal{N}_{x, y}(\boldsymbol{r})$ is the nonlocal steady state transverse conductivity. $b_{J}=\gamma \hbar \mathcal{P}_{0} \sigma_{0} E / 2 e M_{s}$ quantifies the adiabatic torque, while $\alpha_{T}(\boldsymbol{r})=p q \lambda_{E}^{2} \mathcal{N}_{x, y}(\boldsymbol{r})$ and $\beta_{T}(\boldsymbol{r})=p q \lambda_{H}^{2} \mathcal{N}_{x, y}(\boldsymbol{r})$ are dimensionless nonlocal contributions to the Gilbert damping and nonadiabatic torque, respectively. Here $M_{s}$ is the saturation magnetization, and the constants $\lambda_{H}^{2}=\hbar \tilde{P}_{H} \sigma_{H} /\left(2 e \mathcal{P}_{0} \sigma_{0}\right)$ and $\lambda_{E}^{2}=\gamma \hbar^{2} \tilde{P}_{0} \sigma_{0} /\left(4 e^{2} M_{s}\right)$ are length scales associated with the emergent magnetic and electric fields, respectively [21]. In the above expressions, $\mathcal{P}_{0(H)} \equiv \mathcal{P}_{0(H)}^{a}=-\mathcal{P}_{0(H)}^{b}$ is the longitudinal (ordinary Hall) current polarization, where $\mathcal{P}_{0(H)}^{\eta}=$ $\left(\sigma_{0(H)}^{\eta, \uparrow}-\sigma_{0(H)}^{\eta, \downarrow}\right) /\left(\sigma_{0(H)}^{\eta, \uparrow}+\sigma_{0(H)}^{\eta, \downarrow}\right)$. Finally, we also need to define the effective polarizations $\bar{P}_{0, H}=\left(\mathcal{P}_{0(H)}+P_{k}\right) / 2$ and $\tilde{P}_{0(H)}=\left(1+\mathcal{P}_{0(H)} P_{k}\right) / 2[33]$.

Interesting physics of charge and spin transport in AFM Sks can be inferred from Eq. (2). Indeed, since $\eta$ changes sign on different sublattices, there is (i) no macroscopic transverse (along $\mathbf{y}$ ) charge current, i.e., no topological Hall effect (THE) [29,30], (ii) no macroscopic longitudinal

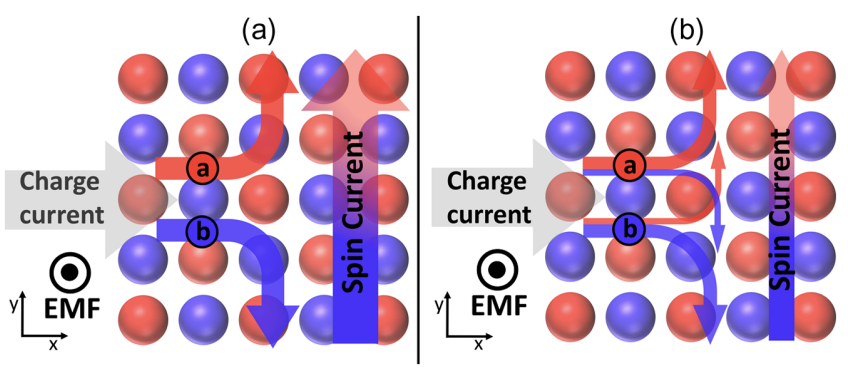

FIG. 1. Schematic illustration of the physical origin of the TSHE in an AFM Sk. (a) For $J \gg t$ and close to the van Hove singularity, two bands are essentially decoupled $\left(P_{k}^{\eta}=\eta\right)$, and the emergent magnetic field (EMF) results to a substantial TSHE. (b) For $J \sim t$ (away from the van Hove singularity), a strong transition between degenerate bands results to a substantial reduction of the TSHE.

(along $\mathbf{x}$ ) spin current, and (iii) a nonzero transverse spin current, i.e., a finite TSHE $[26,42]$. The physical origin of the TSHE as illustrated in Fig. 1 stems from the interplay between the emergent magnetic field, Eq. (1b), and the dispersion of the underlying system. The emergent magnetic field deflects flowing electrons with opposite spins to opposite directions, and the inherent twofold degeneracy ensures that a continuous transverse pure spin current flows in the system.

To elucidate the effect of this transverse spin current on the mobility of Skyrmions, the impact of the topological spin current derived in Eq. (2b) on the dynamics of an AFM Sk is investigated. To achieve this, we calculate the corresponding total spin torque as $\boldsymbol{\tau}_{T}=-\boldsymbol{\nabla} \cdot \boldsymbol{j}_{s}$, where $\boldsymbol{j}_{s}=\boldsymbol{j}_{s}^{a}+\boldsymbol{j}_{s}^{b}$ and, for the sake of completeness, we include nonadiabatic effects [43] via a constant nonadiabaticity $\beta$ and the Gilbert damping torque with damping constant $\alpha$ such that the total spin torque is given as $\boldsymbol{\tau}=\alpha \mathbf{n} \times \partial_{t} \mathbf{n}-$ $\beta b_{J} \mathbf{n} \times \partial_{x} \mathbf{n}-\boldsymbol{\tau}_{T}$ to obtain

$$
\begin{aligned}
\boldsymbol{\tau}= & \alpha \mathbf{n} \times \partial_{t} \mathbf{n}-\beta b_{J} \mathbf{n} \times \partial_{x} \mathbf{n} \\
& +\alpha_{T}(\mathbf{r}) \mathbf{n} \times \partial_{t} \mathbf{n}-\beta_{T}(\mathbf{r}) b_{J} \mathbf{n} \times \partial_{x} \mathbf{n} .
\end{aligned}
$$

It appears clear from Eq. (3) that, just as in FM Sks [20,21], the transverse spin current flowing in AFM Sks directly enhances the nonadiabatic torque and the damping. Moreover, this nonadiabatic topological torque increases when decreasing the Skyrmion size. As a result, the efficiency of the current-driven motion increases when the Skyrmion becomes smaller.

We follow the standard theoretical scheme employed to study the dynamics of antiferromagnetic textures [44-49] supplemented by the derived topological torque to obtain the equation of motion of the Néel order parameter as [33]

$$
\frac{1}{\bar{a} \tilde{\gamma}} \partial_{t}^{2} \mathbf{n}+\alpha_{\mathrm{eff}}(\mathbf{r}) \partial_{t} \mathbf{n}=\gamma \mathbf{f}_{\mathbf{n}}+\beta_{\mathrm{eff}}(\mathbf{r}) b_{J} \partial_{x} \mathbf{n},
$$

where $\tilde{\gamma}=\gamma /\left(1+\alpha^{2}\right), \quad \alpha_{\text {eff }}(\mathbf{r})=\alpha+\alpha_{T}(\mathbf{r}), \quad \beta_{\text {eff }}(\mathbf{r})=$ $\beta+\beta_{T}(\mathbf{r})$, and $\mathbf{f}_{\mathbf{n}}$ is the effective field derived from the 
magnetic energy $E=\int d \mathbf{r}\left[(\bar{a} / 2) \mathbf{m}^{2}+\frac{A}{2}(\nabla \mathbf{n})^{2}\right]$ as $\mathbf{f}_{\mathbf{n}}=$ $-\delta_{\mathbf{n}} E$, where $\bar{a}$ and $A$ are the homogeneous and inhomogeneous exchange constants, respectively [46]. The terminal velocity calculated from Eq. (4) is given as

$$
v_{y}=0 \quad \text { and } \quad v_{x}=\left(\beta_{\text {eff }} / \alpha_{\text {eff }}\right) b_{J},
$$

where the effective parameters are given as [33]

$$
\alpha_{\mathrm{eff}}=\alpha+\frac{4}{3} \frac{\lambda_{E}^{2}}{r_{0}^{2}} \quad \text { and } \quad \beta_{\mathrm{eff}}=\beta+\frac{4}{3} \frac{\lambda_{H}^{2}}{r_{0}^{2}} .
$$

To provide a qualitative estimate of our predicted effect, using realistic material parameters $M_{s}=800 \mathrm{KA} / \mathrm{m}$, $\alpha=0.01, \beta=0.02, P_{0}=0.7, P_{k}=0.4, \sigma_{0}=14.75 /(\mu \Omega \mathrm{m})$, $\sigma_{H} / \sigma_{0}=0.045 / \mathrm{T}$, and $j_{e}=5 \times 10^{11} \mathrm{~A} / \mathrm{m}^{2}$, we obtain $\lambda_{E}^{2}=$ $0.225 \mathrm{~nm}^{2}$ and $\lambda_{H}^{2}=13.54 \mathrm{~nm}^{2}$. These values translate to a longitudinal velocity of up to $391 \mathrm{~m} / \mathrm{s}$ for a Skyrmion size of $10 \mathrm{~nm}$, showing that, for small Skyrmions sizes, the topological torque produces a sizable effect.

Before we proceed, we note that, even though the topological torque discussed above does not rely on SOC, the latter is expected to be ubiquitous in systems promoting noncollinear magnetic textures such as AFM Sks [38-40]. Indeed, SOC has several effects on spin transport, depending on its symmetry. In bulk materials, it contributes to spin relaxation, which results in nonadiabatic torque that gives rise to a Skyrmion mobility that is independent of $r_{0}$ [43]. In magnets lacking inversion symmetry, such as in magnetic multilayers, interfacial (Rashba-like) SOC produces (mostly) a fieldlike torque, while the spin Hall effect arising from an adjacent heavy metal induces a dampinglike torque. The former does not contribute to AFM Sks mobility, while Velkov et al. [28] showed that the latter induces a mobility that is proportional to $r_{0}$. Finally, one also needs to consider the spin Hall effect inside the AFM itself. In the case of ferromagnetic vortices, Manchon and Lee [50] showed that the spin Hall effect acts in the same way as the nonadiabatic torque, thus inducing a mobility that does not depend on the vortex radius. Therefore, we expect the Skyrmion mobility to be dominated by the topological torque discussed above in the limit of small Skyrmions.

Tight-binding model.-Our theoretical predictions in Eqs. (2) and (6) are verified by means of a tight-binding model of an isolated AFM Sk on a square lattice described by the Hamiltonian

$$
\mathcal{H}=\sum_{i} \epsilon_{i} \hat{c}_{i}^{\dagger} \hat{c}_{i}-t \sum_{\langle i j\rangle} \hat{c}_{i}^{\dagger} \hat{c}_{j}-J \sum_{i} \hat{c}_{i}^{\dagger} \mathbf{m}_{i} \cdot \hat{\boldsymbol{\sigma}} \hat{c}_{i},
$$

where $J$ is the exchange energy that couples the spin of electrons $\hat{\boldsymbol{\sigma}}$ to the local magnetization $\mathbf{m}_{i}, t$ is the nearestneighbor hopping, and $\epsilon_{i}$ and $\hat{c}_{i}^{\dagger}\left(\hat{c}_{i}\right)$ are the on-site energy and the spinor creation (annihilation) operator of site $i$, (a)

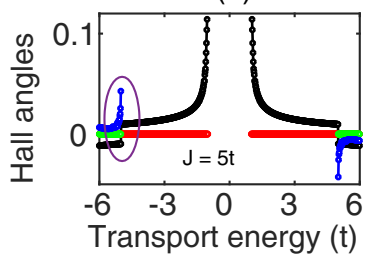

(b)

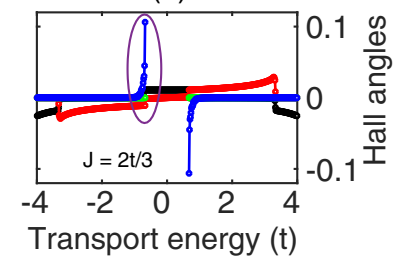

$\rightarrow \theta_{\mathrm{FM}}^{\mathrm{TH}} \rightarrow \theta_{\mathrm{FM}}^{\mathrm{TSH}} \rightarrow \theta_{\mathrm{AFM}}^{\mathrm{TH}} \rightarrow \theta_{\mathrm{AFM}}^{\mathrm{TSH}}$
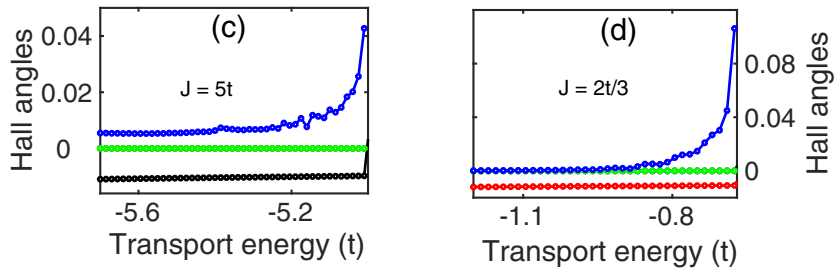

FIG. 2. Computed THE and TSHE for a FM Sk and AFM Sk as a function of the Fermi energy in the (a) strong and (b) intermediate exchange limits. Insets (c) and (d) represent an enlargement around the purple region in (a) and (b), respectively.

respectively. We consider an AFM Sk of radius $12 a_{0}$ to ensure that the texture is smooth and slowly varying [21], for both the strong $(J=5 t)$ and the intermediate $(J=2 t / 3)$ exchange limits [22] using the KWANT code [51]. The Hall transport is investigated via a four-terminal system [23] with a scattering region of size $102 \times 102 a_{0}^{2}$ (i.e., $51 \times 51$ AFM unit cells) and compared with an equivalent FM Sk.

In a clean system, we find a substantial TSHE in AFM Sks in both the strong exchange limit and close to the van Hove singularity [blue line in Figs. 2(a) and 2(b)]. In the intermediate exchange limit, however, the transition between degenerate bands is strong [40]; this results in an overall reduction of the TSHE. Furthermore, since the TSHE increases with the Skyrmion density [23], a substantial spin current capable of inducing magnetization dynamics and/or switching on an adjacent attached FM layer can be expected. Moreover, unlike FM Sks, AFM Sks exhibit no THE due to the cancellation of the charge current contributions from both sublattices [29] [green line in Figs. 2(a) and 2(b)]. Our numerical results are consistent with our analytical predictions in Eq. (2).

To model real materials, we investigated the impact of nonmagnetic impurities which are omnipresent in experiments. This is done via randomized on-site energies $\epsilon_{i}=\mathcal{V}_{i} \in[-(W / 2),(W / 2)]$, where $W$ defines the strength of the disorder, and average over $10^{4}$ configurations to ensure convergence. Two classes of defects are considered: (i) interstitial defects, which preserve the coherence between the sublattices within the antiferromagnetic unit cell, referred to as symmetric scattering (SS), and (ii) disorder that induces decoherence within the unit cell, referred to as asymmetric scattering (AS) [33]. We find that, in both the strong and intermediate exchange limits, as shown in Figs. 3(a) and 3(b), respectively, the presence of disorder 


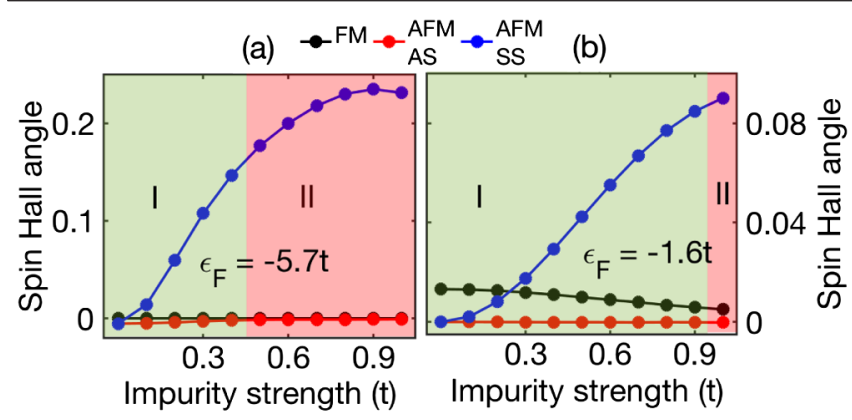

FIG. 3. Dependence of the TSHE on impurity for the (a) strong and (b) intermediate exchange limits. In region I (II), the mean free path of the system is longer (shorter) than the system size. Notice the enhancement of the TSHE in the presence of an impurity for symmetric (blue curve) compared to both asymmetric (red curve) defects and a FM Sk (black curve).

progressively quenches the TSHE for the FM Sk (black curve); in contrast, in the case of the AFM Sk, only AS quenches the TSHE (red curve). In fact, SS disorder enhances the TSHE (blue curve) as long as the coherence between the two sublattices is preserved (region I) $[33,40]$. A further increase in the disorder strength eventually leads to the onset of decoherence (region II), resulting in the reduction of the TSHE.

Finally, we numerically verify the scaling law of the topological torques with respect of the Skyrmion size given by Eq. (3). To achieve this, we follow the scheme outlined in Ref. [21], consider a large system size of $302 \times 302 a_{0}^{2}$, and calculate the local spin transfer torque from the nonequilibrium spin density induced by a voltage bias of $0.2 t$. The calculated torque is then projected on $\partial_{x} \mathbf{n}$ (adiabatic) or $\mathbf{n} \times \partial_{x} \mathbf{n}$ (nonadiabatic), integrated over space, and normalized accordingly to obtain the scaling law with respect to the Skyrmion size. From this, we computed the nonadiabatic torque as $\beta_{\text {eff }} b_{J}=\left[\int \boldsymbol{\tau} \cdot\left(\mathbf{n} \times \partial_{x} \mathbf{n}\right) d^{2} \mathbf{r}\right] / \int \mathcal{N}_{x, y}(\boldsymbol{r}) d^{2} \mathbf{r}[33]$. Our numerical calculations as depicted in Figs. 4(a) and 4(b) show good correspondence with our analytical predictions in Eq. (6) in both the strong and intermediate exchange limits.
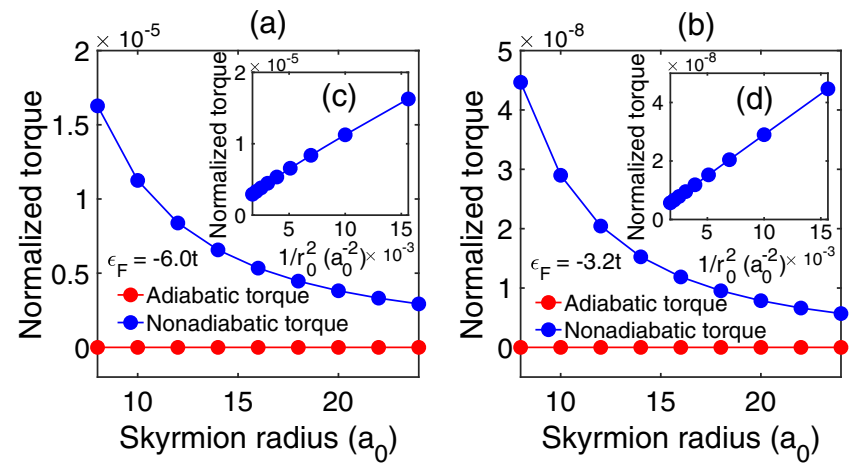

FIG. 4. Dependence of the torques on the Skyrmion radius in the (a) strong and (b) intermediate exchange limits. The insets (c) and (d) show the scaling law of the nonadiabatic torque.
Conclusion.-Micromagnetic simulations originally predicted that Skyrmions have, in principle, limited sensitivity to local and edge defects owing to very weak interactions $[11,12]$ and their finite spatial extension $[15,17,52]$. Indeed, the ability of a defect to pin a Skyrmion increases when the size of the Skyrmion becomes comparable to the size of the defect [11]. Hence, scaling down the Skyrmion towards sub-100-nm size results in low Skyrmion mobility and large critical depinning currents in polycrystalline systems [53]. What makes AFM Sks remarkable in this respect is the fact that the torque efficiency itself increases when reducing the Skyrmion size, as discussed above. While this topological torque contributes only to the transverse motion of FM Sks, it drives the longitudinal motion of AFM Sks and, therefore, directly competes with the enhanced pinning potential. This unique property could be a substantial advantage to compensate the increasing pinning upon size reduction. Furthermore, our calculations show that the TSHE is enhanced in the presence of moderate disorder that is omnipresent in real materials, demonstrating the robustness of the proposed approach for device applications.

This work was supported by Grant-in-Aid for Scientific Research(B) No. 17H02929, from the Japan Society for the Promotion of Science and Grant-in-Aid for Scientific Research on Innovative Areas No. 26103006 from the Ministry of Education, Culture, Sports, Science and Technology (MEXT) of Japan. O. A. T. acknowledges support by the Grants-in-Aid for Scientific Research (No. 25247056, No. 17K05511, and No. 17H05173) from the MEXT of Japan, MaHoJeRo (DAAD Spintronics network, Project No. 57334897), and by JSPS and RFBR under the Japan-Russia Research Cooperative Program. A. M. acknowledges support from the King Abdullah University of Science and Technology (KAUST). C. A. A. thanks R. Cheng for useful discussions.

*collins.akosa@riken.jp

[1] T. Jungwirth, X. Marti, P. Wadley, and J. Wunderlich, Nat. Nanotechnol. 11, 231 (2016).

[2] V. Baltz, A. Manchon, M. Tsoi, T. Moriyama, T. Ono, and T. Tserkovnyak, Rev. Mod. Phys. 90, 015005 (2018).

[3] P. Wadley et al., Science 351, 587 (2016).

[4] S. Fukami, C. Zhang, S. DuttaGupta, A. Kurenkov, and H. Ohno, Nat. Mater. 15, 535 (2016).

[5] A. van den Brink, G. Vermijs, A. Solignac, J. Koo, J. T. Kohlhepp, H. J. M. Swagten, and B. Koopmans, Nat. Commun. 7, 10854 (2016).

[6] C. Sürgers, G. Fischer, P. Winkel, and H. v. Löhneysen, Nat. Commun. 5, 3400 (2014).

[7] S. Nakatsuji, N. Kiyohara, and T. Higo, Nature (London) 527, 212 (2015).

[8] A. K. Nayak et al., Sci. Adv. 2, e1501870 (2016).

[9] O. Gomonay, T. Jungwirth, and J. Sinova, Phys. Rev. Lett. 117, 017202 (2016). 
[10] T. Shiino, S.-H. Oh, P. M. Haney, S.-W. Lee, G. Go, B.-G. Park, and K.-J. Lee, Phys. Rev. Lett. 117, 087203 (2016).

[11] S.-Z. Lin, C. Reichhardt, C. D. Batista, and A. Saxena, Phys. Rev. B 87, 214419 (2013).

[12] C. Reichhardt, D. Ray, and C. J. Olson Reichhardt, Phys. Rev. Lett. 114, 217202 (2015).

[13] S.-Z. Lin, C. Reichhardt, and A. Saxena, Appl. Phys. Lett. 102, 222405 (2013).

[14] J. Iwasaki, M. Mochizuki, and N. Nagaosa, Nat. Commun. 4, 1463 (2013).

[15] J. Iwasaki, M. Mochizuki, and N. Nagaosa, Nat. Nanotechnol. 8, 742 (2013).

[16] J. Iwasaki, W. Koshibae, and N. Nagaosa, Nano Lett. 14, 4432 (2014).

[17] J. Sampaio, V. Cros, S. Rohart, A. Thiaville, and A. Fert, Nat. Nanotechnol. 8, 839 (2013).

[18] K. Everschor, M. Garst, B. Binz, F. Jonietz, S. Muhlbauer, C. Pfleiderer, and A. Rosch, Phys. Rev. B 86, 054432 (2012).

[19] K. Litzius et al., Nat. Phys. 13, 170 (2017).

[20] A. Bisig et al., Phys. Rev. Lett. 117, 277203 (2016).

[21] C. A. Akosa, P. B. Ndiaye, and A. Manchon, Phys. Rev. B 95, 054434 (2017).

[22] G. Yin, Y. Liu, Y. Barlas, J. Zang, and R. K. Lake, Phys. Rev. B 92, 024411 (2015).

[23] P. B. Ndiaye, C. A. Akosa, and A. Manchon, Phys. Rev. B 95, 064426 (2017).

[24] W. Jiang et al., Nat. Phys. 13, 162 (2017).

[25] I. A. Ado, O. A. Tretiakov, and M. Titov, Phys. Rev. B 95, 094401 (2017).

[26] B. Göbel, A. Mook, J. Henk, and I. Mertig, Phys. Rev. B 96, 060406(R) (2017).

[27] C. Jin, C. Song, J. Wang, and Q. Liu, Appl. Phys. Lett. 109, 182404 (2016).

[28] H. Velkov, O. Gomonay, M. Beens, G. Schwiete, A. Brataas, J. Sinova, and R. A. Duine, New J. Phys. 18, 075016 (2016).

[29] J. Barker and O. A. Tretiakov, Phys. Rev. Lett. 116, 147203 (2016).

[30] X. Zhang, Y. Zhou, and M. Ezawa, Sci. Rep. 6, 24795 (2016).

[31] R. Keesman, M. Raaijmakers, A. E. Baerends, G. T. Barkema, and R. A. Duine, Phys. Rev. B 94, 054402 (2016).

[32] H. Fujita and M. Sato, Phys. Rev. B 95, 054421 (2017).
[33] See Supplemental Material at http://link.aps.org/ supplemental/10.1103/PhysRevLett.121.097204 for the calculation details, which includes Refs. [34-37].

[34] C. A. Akosa, W.-S. Kim, A. Bisig, M. Klaui, K.-J. Lee, and A. Manchon, Phys. Rev. B 91, 094411 (2015).

[35] S. E. Barnes and S. Maekawa, Phys. Rev. Lett. 98, 246601 (2007).

[36] Y. Tserkovnyak and M. Mecklenburg, Phys. Rev. B 77, 134407 (2008).

[37] S. Zhang and Steven S.-L. Zhang, Phys. Rev. Lett. 102, 086601 (2009).

[38] Y. Yamane, J. Ieda, and J. Sinova, Phys. Rev. B 93, 180408 (R) (2016).

[39] Y. Yamane, J. Ieda, and J. Sinova, Phys. Rev. B 94, 054409 (2016).

[40] R. Cheng and Q. Niu, Phys. Rev. B 86, 245118 (2012).

[41] Hamed Ben Mohamed Saidaoui, X. Waintal, and A. Manchon, Phys. Rev. B 95, 134424 (2017).

[42] P. M. Buhl, F. Freimuth, S. Blügel, and Y. Mokrousov, Phys. Status Solidi RRL 11, 1700007 (2017).

[43] S. Zhang and Z. Li, Phys. Rev. Lett. 93, 127204 (2004).

[44] R. Cheng and Q. Niu, Phys. Rev. B 89, 081105(R) (2014).

[45] A. C. Swaving and R. A. Duine, Phys. Rev. B 83, 054428 (2011).

[46] K. M. D. Hals, Y. Tserkovnyak, and A. Brataas, Phys. Rev. Lett. 106, 107206 (2011).

[47] A. C. Swaving and R. A. Duine, J. Phys. Condens. Matter 24, 024223 (2012).

[48] E. G. Tveten, A. Qaiumzadeh, O. A. Tretiakov, and A. Brataas, Phys. Rev. Lett. 110, 127208 (2013).

[49] D. R. Rodrigues, K. Everschor-Sitte, O. A. Tretiakov, J. Sinova, and A. Abanov, Phys. Rev. B 95, 174408 (2017).

[50] A. Manchon and K.-J. Lee, Appl. Phys. Lett. 99, 022504 (2011).

[51] C. W. Groth, M. Wimmer, A. R. Akhmerov, and X. Waintal, New J. Phys. 16, 063065 (2014).

[52] R. Tomasello, V Puliafito, E Martinez, A Manchon, M Ricci, M Carpentieri, and G Finocchio, J. Phys. D 50, 325302 (2017).

[53] W. Legrand, D. Maccariello, N. Reyren, K. Garcia, C. Moutafis, C. Moreau-Luchaire, S. Collin, K. Bouzehouane, V. Cros, and A. Fert, Nano Lett. 17, 2703 (2017). 